\section{Vorhersage des Rezidivrisikos beim Kolonkarzinom}

Um die Therapie von Patienten mit Kolonkarzinom zu individualisieren, sind Kenntnisse der Tumorbiologie notwendig. Mit verschiedenen GenAssays versucht man, diesem Ziel ein wenig näher zu kommen.

$Z^{\mathrm{s}}$ ur Auswertung von Multi-Gen-Assays wurde ein Risikoscore entwickelt, der das Rezidivrisiko von Patienten mit Kolonkarzinom vorhersagen soll. Die vorliegende Studie diente der Validierung eines 12-Gen-RezidivScores. Dieser wird anhand der Expression von zwölf Genen (sieben RezidivGene und fünf Referenz-Gene) berechnet. Die Validierung wurde an Formalin-fixierten und in Paraffin eingebetteten Tumorbiopsien von Patienten aus der Studie 9581 der Cancer and Leukemia Group B (CALGB) vorgenommen. In CALGB 9581 waren insgesamt 1.713 Patienten mit einem Kolonkarzinom im Stadium II randomisiert der Behandlung mit Edrecolomab oder der alleinigen Beobachtung zugeteilt worden.
Dabei wurde kein Unterschied im Überleben beobachtet. Ausgewertet wurden nun die Tumorbiopsien von 690 Patienten, von denen 162 ein Rezidiv hatten.

Es zeigte sich, dass der 12-Gen-Rezidiv-Score signifikant mit dem Rezidivrisiko assoziiert war $(p=0,013)$. Von den konventionellen klinischen und pathologischen Faktoren war ein Gendefekt im Mismatch-Reparatur-System (MMR) der einzige Faktor, der ebenfalls signifikant mit dem Rezidivrisiko assoziiert war $(\mathrm{p}=0,044)$.

In multivariaten Analysen war der 12-Gen-Rezidiv-Score der stärkste Prädiktor für ein Rezidiv ( $p=0,004)$, und zwar unabhängig von der Tumorgröße, vom MMR-Mutationsstatus, der Anzahl der untersuchten Lymphknoten, dem Tumorgrading und der Ausbreitung in die Lymphgefäße.

Der 12-Gen-Rezidiv-Score scheint die besten Vorhersagen bei Patienten mit einem T3-Tumor und intakten MMR-Genen (MMR-I) zu liefern. Für diese Patienten ergaben sich beispielsweise folgende Rezidivrisiken: Patienten mit einem niedrigen 12-Gen-Rezidiv-Score hatten ein durchschnittliches 5-Jahres-Rezidivrisiko von $13 \%$, bei Patienten mit mittlerem 12-Gen-RezidivScore betrug dieses Risiko $16 \%$ und bei Patienten mit hohem 12-Gen-RezidivScore $21 \%$.

Fazit: Mithilfe des 12-Gen-RezidivScores konnte das Rezidivrisiko von Patienten mit einem Kolonkarzinom im Stadium II bestimmt werden.

\section{Judith Neumaier}

Venook AP et al. Biologic Determinants of tumor recurrence in stage II colon cancer: Validation study of the 12-gene recurrence score in Cancer and Leukemia Group B (CALGB) 9581. J Clin Oncol. 2013;31(14):1775-81.

\title{
TKI-Kombination bei mCRC enttäuscht
}

Der monoklonale Antikörper Cetuximab hat das Überleben von Patienten mit metastasiertem kolorektalem Karzinom (mCRC) und KRAS-Wildtyp verbessert. In einer Phase-III-Studie wurde untersucht, ob die Kombination mit dem Multi-Tyrosinkinaseinhibitor (TKI) Brivanib das Ergebnis weiter verbessert.

B rivanib hemmt die Rezeptoren für vaskulären endothelialen Wachstumsfaktor (VEGF) und FibroblastenWachstumsfaktor (FGFR). Erste klinische Studien beim mCRC hatten eine

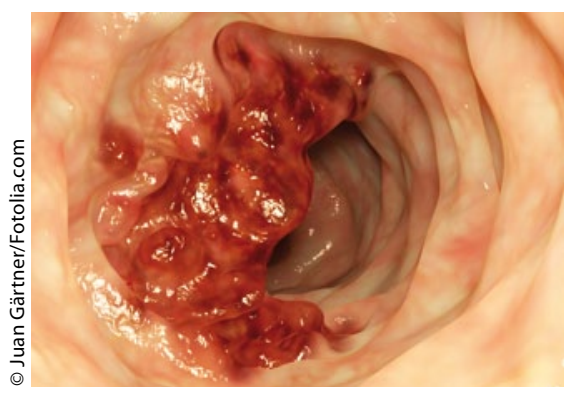

Etwa $35 \%$ aller Patienten mit CRC weisen bereits bei der Erstdiagnose Metastasen auf. vielversprechende Aktivität der Kombination mit Cetuximab gezeigt. An der aktuellen Studie nahmen 750 Patienten mit mCRC teil, die zuvor bereits eine Kombinationschemotherapie erhalten hatten. Randomisiert erhielten sie zusätzlich zu Cetuximab entweder Brivanib (täglich 800 mg oral, Arm A, n = 376) oder Placebo (Arm B, $\mathrm{n}=374$ ).

Beim primären Endpunkt, dem medianen Gesamtüberleben (OS), unterschieden sich nach median 18,7 Monaten die Studienarme nicht signifikant: 8,8 Monate in Arm A und 8,1 Monate in Arm B (Hazard Ratio [HR] 0,88; 95\%-KI 0,741,03 ; $\mathrm{p}=0,12$ ). Das mediane progressionsfreie Überleben (PFS) war dagegen im Interventionsarm mit 5,0 Monaten signifikant höher als im Kontrollarm mit 3,4
Monaten $(\mathrm{HR}=0,72 ; 95 \%-\mathrm{KI}=0,62-$ $0,84 ; \mathrm{p}<0,001)$, und auch der Anteil von Patienten mit partiellem Ansprechen war höher (13,6 vs. 7,2\%; p = 0,004). Die Kombination erhöhte allerdings auch die Inzidenz von Nebenwirkungen vom Grad $\geq 3$ (78 vs. $53 \%$ ). Dabei war der Anteil der Patienten, der die geplante Dosisintensität erhalten hatte, im Kombinationsarm deutlich niedriger: Nur $57 \%$ der Patienten erhielten hier $\geq 90 \%$ der geplanten Dosisintensität von Cetuximab, im Kontrollarm waren es $83 \%$.

Fazit: PFS und Ansprechen fielen in dieser Studie zwar zugunsten der Kombination von Cetuximab und Brivanib aus. Dies resultierte aber nicht in einem besseren Gesamtüberleben und war mit höherer Toxizität verbunden. Friederike Klein

Siu LL et al. Phase III randomized, placebocontrolled study of cetuximab plus brivanib alaninate versus cetuximab plus placebo in patients with metastatic, chemotherapyrefractory, wild-type K-RAS colorectal carcinoma: The NCIC Clinical Trials Group and AGITG CO.20 trial. J Clin Oncol. 2013.31(19):2477-84. 